\title{
Anticipating partners' responses: Examining item and source memory following interactive exchanges
}

\author{
MARY ANN FOLEY and HUGH J. FOLEY \\ Skidmore College, Saratoga Springs, New York \\ JAIME R. DURLEY \\ University of Georgia, Athens, Georgia \\ and \\ ANGELA T. MAITNER \\ University of California, Santa Barbara, California
}

\begin{abstract}
Within the context of an interactive anagram-solving task, the present studies tested predictions about the role of cognitive anticipation in both source and item memory. After working in pairs to solve anagram problems, participants were surprised by a source-monitoring test focused on the source of solutions (self vs. partner, Experiment 1) or a standard recognition test focused on the solutions themselves (Experiment 2). With the intention of affecting the opportunity to anticipate partners' solutions, two variables were manipulated: anagram difficulty (easy vs. hard) and the delay between the presentation of an anagram problem and the prompt that designated one member of each pair as the anagram solver. Consistent with predictions, as the opportunity to anticipate partners' solutions increased, there was a decrease in source accuracy suggesting increased confusion about whether the solution had been self- or partner-generated. Generation-effect failures were observed in item memory. However, these failures reflected increases in item memory for partners' responses rather than decreases in memory for self-generated ones. These studies suggest that when opportunities to anticipate partners' responses are available, self-generative activities may be associated with both selfand partner-generated items, influencing the expression of the generation effect.
\end{abstract}

Advantages of self-generated information for memory have been noted for well over two decades (see, e.g., Johnson, Raye, Foley, \& Foley, 1981; Mulligan, 2001; Slamecka \& Graf, 1978). In a prototypical study revealing these advantages, individuals generate materials following various kinds of encoding rules while also encoding materials provided by someone else. On subsequent memory tests, self-generated materials are often better recognized and recalled than the control materials provided by someone else. Furthermore, the self-generated materials are identified as such by the individual, showing advantages for both item and source memory (e.g., Foley \& Ratner, 1998; Johnson et al., 1981; Kinjo \& Snodgrass, 2000; R. L. Marsh \& Hicks, 1998, Experiment 1).

This research was supported by NSF/REU Grant SBR-9619404 to the first two authors. The authors thank Elizabeth Marsh and two anonymous reviewers for helpful comments on earlier versions of this article. Correspondence concerning this article should be sent to M. A. Foley, Department of Psychology, Skidmore College, Saratoga Springs, NY 12866 (e-mail: mfoley@skidmore.edu).

Note-This article was accepted by the previous editorial team, when Colin M. MacLeod was Editor.
The beneficial effects of generating are robust for both item and source memory (for reviews, see Greene, 1992; Mulligan, 2001, 2004; Steffens \& Erdfelder, 1998; Taconnat \& Isingrini, 2004). Numerous demonstrations of these effects are documented across a variety of encoding (Foley \& Foley, in press; Foley, Foley, Wilder, \& Rusch, 1989; Johnson et al., 1981; Mulligan, 2001, 2004; Slamecka \& Graf, 1978) and test (R. L. Marsh \& Hicks, 1998; Srinivas \& Roediger, 1990, Experiment 1) conditions. Moreover, they are maintained across relatively long retention intervals for both item memory (e.g., 7 days; Gardiner, Ramponi, \& Richardson-Klavehn, 1999) and source memory (e.g., 10 days; Johnson et al., 1981).

In most investigations of the generation effect, experimenters are the source of control materials (e.g., Johnson et al., 1981; Mulligan, 2001; Slamecka \& Graf, 1978). However, other individuals (in particular, partners who are involved in their own acts of generating as part of interactive exchanges) may also be the source of control materials (Baker-Ward, Hess, \& Flannagan, 1990; Foley \& Ratner, 1998; Jurica \& Shimamura, 1999). The present studies were designed to investigate item and source memory in such collaborative exchanges, because in these contexts the generation effect may not be observed. 
For example, after asking child triads to take turns performing and observing a series of play activities (e.g., playing a song on a xylophone, bouncing tennis balls into a trash can), Baker-Ward et al. (1990) reported that child participants recalled more "self" than "partner" actions. When friends were members of the triads, however, a generation effect was not observed. What is most interesting to us is that the disappearance of the generation effect resulted from a relative increase in the recall of partners' actions. In two studies, recall of self-actions was relatively good and constant, with the recall of partner's actions comparable to that of self-actions only when the other participants in the triads were friends (Baker-Ward et al., 1990).

Similar generation-effect "failures" have been reported in other contexts, including those in which the members of interactive pairs were not friends but worked interactively to complete a common goal (Foley \& Ratner, 1998; Foley, Ratner, \& House, 2002). For example, in a source-monitoring task, when asked to remember who contributed what to the completion of a shared, goaldirected activity, young children take undue credit for others' contributions, falsely reporting that they have performed actions that were actually performed by their partners (Foley \& Ratner, 1998; Foley, Ratner, \& Gentes, 2005; Foley, Ratner, \& Passalacqua, 1993). We will refer to these errors in source monitoring as self-errors. Documented in over 25 studies, self-errors are observed for a number of interactive exchanges, including collage making (Foley et al., 1993), search tasks modeled on the Where's Waldo? children's book (Foley et al., 2002), and a dollhouse construction task (Ratner, Foley, \& Gimpert, 2002). These self-errors, however, did not reflect worse item-memory for partners' contributions than for self-contributions, as would define a generation effect. Rather, when examined within the context of source-monitoring judgments or independently by way of recognition tests, item memory for self- and partner actions was quite good and comparable (Foley \& Ratner, 1998; Foley et al., 2002, Experiment 3). Thus, increased self-errors may be associated with item generation-effect failures.

The tendency of children to take credit for the contributions of others is thought to reflect recoding errors that occur during encoding. According to this view (Foley et al., 1993), while waiting for a partner to complete an action, a child may anticipate the partner's response and later confuse the source of the contribution; that is, the child may remember the anticipation as the actual contribution. Consistent with this emphasis on the role of anticipation, when the opportunity to anticipate is precluded (Foley et al., 2002) or manipulated by way of instructions (Foley \& Ratner, 1998) or variations in turn taking (Foley et al., 2002), self-errors are affected in predictable ways. Moreover, the timing of the partner's responses in relation to those of the child is of critical importance (Foley et al., 2002). If turns are not alternated but occur in blocks, such that children watch while their partners complete all of their turns first, the tendency to take undue credit is observed. However, when turns are blocked so that children complete all of their tasks before their partners have an opportunity to do so, the self-error is not observed.

Resistance to these kinds of self-errors may increase with age, but the similar tendencies of adults to take undue credit for the contributions of others have been evident in the literature for many years (see, e.g., Foley \& Ratner, 1998; Greenwald, 1980; Macrae, Bodenhausen, \& Calvini, 1999). For example, in studies of inadvertent plagiarism, despite specific instructions to avoid repetition, adults often repeat their partners' responses as though they have not been previously generated (e.g., Brown \& Murphy, 1989; Landau \& Marsh, 1997; E. J. Marsh, Edelman, \& Bower, 2001; R. L. Marsh \& Bower, 1993; R. L. Marsh, Landau, \& Hicks, 1997; Taylor, 1965). These error patterns are consistent with the suggestion that as participants await their next turn, self-anticipation may occur and possibly influence subsequent performance (Foley \& Ratner, 1998; Johnson, Hashtroudi, \& Lindsay, 1993). When an experimenter instructs adult participants to guess their partners' solutions to word problems (Landau \& Marsh, 1997), acts of inadvertent plagiarism increase. Similarly, when turn taking occurs in a blocked rather than in an alternating manner, individuals who wait for their partners to complete all of their responses before beginning their turn are far more likely to commit inadvertent acts of plagiarism, repeating their partners' responses from the first phase of the experiment. Indeed, levels of inadvertent plagiarism are particularly pronounced under these blocking conditions (R. L. Marsh \& Landau, 1995).

Thus, the study of memory for collaborative exchanges in both children (Foley \& Ratner, 1998; Foley et al., 2002) and adults (e.g., Greenwald, 1980; R. L. Marsh \& Bower, 1993 ) suggests that memory accuracy is sensitive to anticipation, leading us to expect that anticipation may contribute to apparent generation-effect failures. A second kind of encoding process may also contribute to source errors and generation-effect failures in these contexts. After observing the partner's responses, an individual could recode those observations as self-actions. The individual would later exhibit confusion about who produced particular responses, but would remember quite well the responses that were actually generated by those participating in the exchange. To date, no research has focused on separating the potentially independent effects of these two kinds of recoding processes - anticipation and observation. In the present experiments, by precluding the opportunity to anticipate under some conditions but allowing participants to hear their partners' responses, we seek to identify the potential role of these two kinds of cognitive operations on item and source memory.

Using an anagram-solving task in which adults working in pairs took turns solving a set of problems, we examined the possible contribution of anticipating and recoding a partner's responses as one's own. An anagram-solving task was selected for several reasons. The reports of generationeffect failures of greatest interest to us are those occurring within the context of interactive exchanges between partners or members of triads. An anagram task is well suited 
for creating these interactive exchanges. Because the task was described to participant pairs as an opportunity for their team to win a modest monetary award for best overall performance, we conceptualized this exchange as somewhat collaborative as well. Most important, the use of an anagram task also allowed manipulation of the opportunity to anticipate a partner's responses.

In the problem-solving phase of the study, participants took on one of two roles on a given trial. Although they could not predict the role they would play, they knew that they would be called on either to solve an anagram or to work on a number-search task. The likelihood of anticipating anagram solutions by the nonsolving partner was manipulated in two ways. First, on some trials, the nonsolving partner never saw the to-be-solved anagram, so no anticipation was possible. The nonsolving partner, however, did hear the solution reported out loud at the end of each trial and thus could recode the solution as self-generated. On other trials, the anagram was visible one of two delay times ( 5 or $15 \mathrm{sec}$ ), which allowed both participants to solve the anagram before learning which one of them was responsible for the solution on that particular trial. This delay manipulation was between groups. Second, participants saw two types of anagrams (easy vs. hard) throughout this phase of the study. The likelihood of correctly anticipating the anagram solution was greater with easy anagrams.

After completing the anagram-solving phase, participants were asked to remember the words generated as solutions (an item memory test; Experiments 1 and 2) or the source of those solutions (self vs. partner; Experiment 1). Thus, item memory was assessed within the context of source judgment in Experiment 1 and by way of a standard recognition test without a source-judgment task in Experiment 2. We expected that the opportunity to anticipate responses would differentially affect source and item memory.

Predictions about the effects of anticipation on memory for self- and partner-generated solutions differed for source memory and item memory. We predicted that increases in the opportunity to anticipate partners' solutions would increase confusion about who generated particular solutions, thereby decreasing source accuracy. In the two delay conditions in Experiment 1, if individuals anticipated at least some of the partner's responses, then partner- and self-items would both be functionally self-generated ones. Under these conditions, there should be considerable confusion about who generated which solutions. In the 0 -sec delay condition, when no anticipation of the solution was possible, we expected source accuracy to be better than in the two delay conditions. If recoding of source took place, however, hearing partners generate their responses in the absence of the opportunity to anticipate would lead to source confusion in this condition as well. The manipulation of the anagram difficulty was expected to affect the likelihood of anticipating partners' responses, resulting in fewer anticipations accompanying hard anagrams. Therefore, we also expected source accuracy to be affected by this variable, resulting in greater source accuracy for solutions to hard anagrams than to easy anagrams.

In contrast to its negative effects on source accuracy, cognitive anticipation was expected to have a facilitative effect on item memory. Because increasing delay also increases the opportunity to anticipate materials generated by one's partner (control materials for assessing the presence of a generation effect), we predicted an increase in memory for partners' solutions (serving as the nongenerated control materials) as a function of delay. If individuals anticipated their partner's responses, then the cognitive operations associated with both self- and partner-generated solutions should enhance item memory, leading to a decrease or elimination of the generation effect at longer delays. Specifically, in both Experiments 1 and 2, we expected to observe the standard generation effect for item (solution) memory in the 0 -sec delay condition when no anticipation was possible, but a decrease in the generation effect for item memory in the 5- and 15-sec delay conditions when anticipation was possible. That is, we predicted a delay $X$ source interaction.

\section{EXPERIMENT 1}

\section{Method}

Participants. Sixty undergraduates attending a liberal arts college were recruited and paid $\$ 5.00$ for their participation.

Materials. Eighty high-frequency words were used (all A or AA from the Thorndike \& Lorge, 1944, norms), each containing an average of seven letters. Anagrams were selected from sets used in previous studies (Foley \& Foley, in press; Foley et al., 1989). Easy and hard anagram versions were constructed for each word using the guidelines described for other studies in this series (see, e.g., Mayzner \& Tresselt, 1958). The first letter of each anagram was also the first letter of its solution, and all words except for one had a single solution. With this first-letter constraint, an easy anagram was defined as one in which the letter order was maximally similar to its word solution, and a hard anagram was defined as one in which the letter order was quite different from the ordering of the letters in the word. In comparison with baseline conditions reported in our previous studies (e.g., simply repeating words), these anagram materials produce a positive generation effect; that is, words generated as solutions are remembered better than nongenerated control words that are read (Foley et al., 1989). A different group of undergraduates $(n=20)$ solved the anagrams, providing norms for those problems included in this study. The hard anagrams took longer to solve; solution times were 4.31 and $8.87 \mathrm{sec}$ for easy and hard anagrams, respectively.

Two sets of 40 items were constructed - one target and one distractor. For the set of 40 targets, four lists were constructed with an item assigned to easy/self, difficult/self, easy/partner, and difficult/partner across lists. Eight practice items were selected from the same set of norms. The 40 word solutions were combined with 40 distractor words for the surprise memory test.

Apparatus. Two Macintosh computers running SuperLab were used to present the anagrams and test materials. The anagramsolving task was administered on a single computer screen and solution times were recorded automatically. A second computer was also set up so that the source-monitoring tests were administered simultaneously to each participant in the pair.

Procedure. Participants, tested in pairs, were told that the study was about teamwork and problem solving. To make this cover story 
more convincing, they were also told that the team with the best overall score (defined in terms of speed and accuracy) on both the anagram and number-search tasks would earn $\$ 25.00$. Participants were informed that the problem-solving task involved taking turns at solving anagrams that would be presented on a computer screen. The anagram to be solved was presented to both participants simultaneously on the left and right sides of the computer screen, respectively. A barrier prevented an individual from seeing the partner's information. Participants were told that the computer would provide a cue on each problem trial that would identify whose turn it was to solve that problem. If there was no delay between the presentation of the anagram and the prompt directing someone to solve it ( 0 -sec condition), one person saw the cue "Solve Anagram" and an anagram, and the other person saw the cue "Work on Number Search," but no anagram. The person receiving the "Number Search" prompt began working on a sheet of paper that displayed multiple number sequences. This number-search task was selected from Games magazine for its difficulty and attention-grabbing characteristics. The person working on this number-search task continued to search for number strings until the partner said his or her word solution aloud.

Participant pairs, arbitrarily created by scheduling constraints, were randomly assigned to one of the three conditions that were defined in terms of the amount of time between the presentation of the anagram and the prompt that designated whose turn it was to solve it. The prompt occurred immediately ( 0 -sec delay condition), $5 \mathrm{sec}$ after the anagram was presented, or $15 \mathrm{sec}$ after the anagram was presented. When waiting for a prompt identifying the person to solve a particular anagram, participants were encouraged to try to figure out the solution. It was assumed that both participants were more likely to solve the anagram with longer delays between its presentation and the prompt.

In the 0 -sec delay condition, one member of the pair was given a prompt to solve the anagram. The other member of the pair was given a prompt to begin searching for number strings in a number-search task (intended as filler) until his or her partner solved the anagram. Once the partner who had received the "Solve Anagram" prompt had an answer, he or she raised a hand and said the solution aloud after the other person turned the number-search task face down. If the solution was correct, the experimenter prompted the computer to present the next problem. If the solution was incorrect, the pair resumed their respective activities (solving the anagram or searching for numbers). In the remaining two conditions, participants were given the same instructions, but in these cases the prompts for the participants ("Solve Anagram" or "Begin Number Search") were presented 5 or $15 \mathrm{sec}$ after the anagram itself.

In all three conditions, if a participant did not solve an anagram within $30 \mathrm{sec}$, he or she was given the second letter of the anagram solution. This additional prompting did not occur often (only $5 \%$ of the participants on one or two anagram trials). Ultimately, all participants were able to solve all anagrams. Failure to follow directions, such as blurting out the solutions at inappropriate times, did not occur.

After completion of the problem-solving phase and a 5-min retention interval during which participants worked on spatial mazes that were also selected from Games magazine, participants were surprised with a source-monitoring test, which was administered separately in adjoining rooms. Each room contained a computer that presented 80 words on its screen, ensuring that participants were unaware of each other's responses. For each test item, participants indicated whether they thought the word had been a solution to an anagram that they had solved, that their partner had solved, or if it was a new word not included in the problem set.

\section{Results}

Item memory. Item memory was computed as the proportion of old items to which a "self" or "partner" choice was made during the source-judgment task. The mean
Table 1

Proportion of Old Items Called "Old" (Item Memory) in Experiment 1 as a Function of Delay, Source, and Anagram Difficulty

\begin{tabular}{|c|c|c|c|c|c|c|}
\hline \multirow{3}{*}{$\begin{array}{l}\text { Anagram } \\
\text { Difficulty }\end{array}$} & \multicolumn{6}{|c|}{ Delay } \\
\hline & \multicolumn{2}{|c|}{$0 \mathrm{sec}$} & \multicolumn{2}{|c|}{$5 \mathrm{sec}$} & \multicolumn{2}{|c|}{$15 \mathrm{sec}$} \\
\hline & $M$ & $S E$ & $M$ & $S E$ & $M$ & $S E$ \\
\hline \multicolumn{7}{|c|}{ Self-Generated } \\
\hline Easy & .80 & .03 & .85 & .03 & .86 & .02 \\
\hline Hard & .90 & .02 & .85 & .03 & .88 & .02 \\
\hline \multicolumn{7}{|c|}{ Partner-Generated } \\
\hline Easy & .55 & .04 & .72 & .04 & .85 & .03 \\
\hline Hard & .57 & .05 & .73 & .03 & .88 & .02 \\
\hline
\end{tabular}

proportions are reported in Table 1 as a function of source of solution (self or partner), anagram difficulty (easy or hard), and delay $(0,5$, or $15 \mathrm{sec})$. An ANOVA showed four significant effects. There was a main effect of anagram difficulty $\left[F(1,69)=4.23, M S_{\mathrm{e}}=.15, p=.04\right]$, in which item memory was better for solutions to hard anagrams $(M=.80)$ than to easy ones $(M=.77)$. There was a main effect of delay $\left[F(2,69)=10.07, M S_{\mathrm{e}}=.63, p<.001\right]$, with increased item memory associated with longer delays. There was a main effect of source $[F(1,69)=89.32$, $\left.M S_{\mathrm{e}}=.15, p<.001\right]$, replicating the standard generation effect with better memory for self-generated items $(M=$ $.86)$ than for partner-generated items $(M=.72)$. However, as predicted, there was a source $\times$ delay interaction $\left[F(1,69)=32.49, M S_{\mathrm{e}}=.15, p<.001\right]$. Tukey-Kramer post hoc tests showed that item memory for self-generated items was better than item memory for partner-generated items at the two shorter delays $(0 \mathrm{sec}$ and $5 \mathrm{sec})$, but item memory for self- and partner-generated items did not differ at the $15 \mathrm{sec}$ delay. As predicted, the generation effect decreased as delay increased because of an increase in item memory for partner's solutions.

False positives. The proportion of new items called old (false positives) was computed as the proportion of new items to which an incorrect "self" or "partner" response was made during the source-judgment task. The mean proportions are reported in Table 2 as a function of delay $(0,5$, or $15 \mathrm{sec})$ and incorrect source choice (self or partner). An ANOVA showed a main effect of delay $\left[F(2,69)=6.54, M S_{\mathrm{e}}=.15, p=.002\right]$, with decreases in false positives associated with longer delays. There was a main effect of incorrect source choice $[F(1,69)=55.58$, $\left.M S_{\mathrm{e}}=.008, p<.001\right]$, with more incorrect "partner" responses $(M=.12)$ than "self" responses $(M=.03)$. There was also a delay $\times$ incorrect source choice interaction $\left[F(2,69)=5.75, M S_{\mathrm{e}}=.008, p<.005\right]$. The simple effect of delay for "self" choices was not significant. However, the simple effect of delay for "partner" choices was significant: "Partner" false positives reliably decreased with an increase in delay. This pattern in the false positives rules out the possibility that the increase in partner item memory over delay was due to an increased bias toward reporting partner. The probability of making a "partner" 
Table 2

Proportion of New Items Called "Old" (False Positives) in Experiments 1 and 2 as a Function of Delay and Incorrect Source Choice

\begin{tabular}{|c|c|c|c|c|c|c|}
\hline \multirow{3}{*}{$\begin{array}{c}\text { Incorrect } \\
\text { Source Choice } \\
\end{array}$} & \multicolumn{6}{|c|}{ Delay } \\
\hline & \multicolumn{2}{|c|}{$0 \mathrm{sec}$} & \multicolumn{2}{|c|}{$5 \mathrm{sec}$} & \multicolumn{2}{|c|}{$15 \mathrm{sec}$} \\
\hline & $M$ & $S E$ & $M$ & $S E$ & $M$ & $S E$ \\
\hline \multicolumn{7}{|c|}{ Experiment 1} \\
\hline "Self" & .05 & .05 & .03 & .03 & .02 & .03 \\
\hline "Partner" & .20 & .10 & .11 & .09 & .07 & .08 \\
\hline \multicolumn{7}{|c|}{ Experiment 2} \\
\hline "Old" & .17 & .01 & .14 & .01 & .09 & .01 \\
\hline
\end{tabular}

false positive reliably decreased with an increase in delay, so it cannot explain a reliable increase in correct recognition of partner items with an increase in delay.

Source accuracy. Correct source judgment (source accuracy) was computed as the number of correct source choices made during the source-monitoring task (e.g., responding "I did" to solutions generated by the self) divided by the number of self-generated solutions to which a "self" or "partner" choice was made (e.g., responding either "I did" or "partner did" in classifying the source of word solutions). The source accuracy scores are reported in Table 3 as a function of source of solution (self or partner), anagram difficulty (easy or hard), and delay $(0,5$, or $15 \mathrm{sec}$ ). An ANOVA showed two significant effects. There was a main effect of anagram difficulty $[F(1,69)=17.96$, $\left.M S_{\mathrm{e}}=.02, p<.001\right]$ in which source accuracy was better for hard anagram solutions $(M=.82)$ than for easy anagram solutions $(M=.75)$. There was also an anagram difficulty $\times$ delay interaction $\left[F(2,69)=4.65, M S_{\mathrm{e}}=\right.$ $.02, p=.01]$. Tukey-Kramer post hoc tests showed that source accuracy did not vary significantly across the delay conditions for solutions to hard anagrams $(M \mathrm{~s}=.82, .81$, and .83 , respectively). However, for the solutions to easy anagrams, decrease in source accuracy was associated with increase in delay $(M \mathrm{~s}=.82, .73$, and .70). Source accuracy was comparable for self- and partner solutions, and there were no interactions involving this variable.

\section{Discussion}

The results of Experiment 1 indicate that controlling the opportunity to anticipate partners' responses affects both source and item memory, but in different and predicted ways. Source accuracy decreased with increases in the delay between the presentation of an anagram and the designation of who was responsible for generating the solution. This decrease, however, was specific to easy anagram solutions. Presumably, anticipations associated with easy anagrams are relatively automatic, leading to their quick resolution. Because the resemblance between anagram letter arrangements and their solutions affects the nature of processing invoked during encoding (Srinivas \& Roediger, 1990), we expected easy anagrams to be solved or noticed right away, thereby making the underlying processes relatively automatic and resulting in the word so- lutions. In contrast, for difficult anagrams, the attempts to solve (and corresponding anticipations) were expected to be more deliberate, subsequently aiding in decisions about anagram source. Remembering attempts to produce a solution, or feeling thwarted when a successful attempt was aborted, could later help render correct source decisions as well. Because we expected anticipation to occur for "self" and "partner" items in the two delay conditions, we correctly expected accuracy to be similar for these two item types.

The dissociation between source and item memory that was predicted and observed in Experiment 1 is also consistent with our emphasis on the role of cognitive anticipation. Although source accuracy decreased with increases in delay, item memory increased. However, this increase was expressed as a generation-effect failure because the increase was attributable to an increase in memory for the partner's responses.

The false positive findings are important for ruling out an alternative explanation for our item and source effects. A bias toward reporting "partner" when in doubt could lead to an artificial inflation of item memory for partnergenerated items. If a participant knew that an item occurred during the problem-solving phase but was uncertain about the source of the item, he or she might use the response "partner" as a default. If this had been the case, we should have observed a tendency to report "partner" when the participant thought that a new item had been seen before. (This expectation is based on the assumption that a bias toward reporting "partner" would operate on responses to both old and new items.) Although there was evidence for this kind of bias under the 0-sec delay condition, as delay increased the incidences of "partner" misreports decreased considerably. Thus, the item-memory advantage for partner-generated items in the two delay conditions is not produced by a bias toward reporting "partner." Along similar lines, recognition of self-generated items did not vary across delays - a pattern that would be expected if a bias toward reporting "partner" increased with increases across delays.

The pattern of findings for the false positive data also rules out an alternative explanation for the source accuracy findings. If the bias toward reporting "partner" increased

Table 3

Proportion of Correct Source Judgments for Old Items (Source Accuracy) in Experiment 1 as a Function of Delay, Source, and Anagram Difficulty

\begin{tabular}{|c|c|c|c|c|c|c|}
\hline \multirow{3}{*}{$\begin{array}{l}\text { Anagram } \\
\text { Difficulty }\end{array}$} & \multicolumn{6}{|c|}{ Delay } \\
\hline & \multicolumn{2}{|c|}{$0 \mathrm{sec}$} & \multicolumn{2}{|c|}{$5 \mathrm{sec}$} & \multicolumn{2}{|c|}{$15 \mathrm{sec}$} \\
\hline & $M$ & $S E$ & $M$ & $S E$ & $M$ & $S E$ \\
\hline \multicolumn{7}{|c|}{ Self-Generated } \\
\hline Easy & .80 & .03 & .73 & .03 & .68 & .04 \\
\hline Hard & .84 & .03 & .77 & .02 & .84 & .03 \\
\hline \multicolumn{7}{|c|}{ Partner-Generated } \\
\hline Easy & .84 & .06 & .72 & .04 & .71 & .04 \\
\hline Hard & .80 & .04 & .84 & .03 & .81 & .03 \\
\hline
\end{tabular}


with increases in delay, then the decrease in source accuracy that we observed could reflect an increasing tendency to report "partner" when in doubt. Again, such an argument fails because the bias toward reporting "partner" decreased with increases in delay. Before further discussing the significance of the dissociation between item and source memory, we look to see whether the pattern observed for item memory was observed again in a second experiment in which recognition judgments were assessed independently from source judgments.

In Experiment 1, item memory was assessed indirectly from source-monitoring scores. Although item and source memory can be affected in similar ways by encoding and test manipulations, this is not always the case (Lindsay \& Johnson, 1991). Furthermore, dissociations between solution and source memory have been reported for other kinds of source judgments about anagram solving (Foley $\&$ Foley, in press). In fact, memory errors are sometimes worse when assessed by way of source judgments rather than standard recognition judgments (Hicks \& Marsh, 2001). Others have argued that the way that item memory is assessed in the context of source judgments (based on recalculations of source scores) may contaminate their assessment (Bayen, Murnane, \& Erdfelder, 1996; Murnane \& Bayen, 1996). Although in other anagram studies we have shown that item memory is equivalent whether or not it is derived from source-monitoring judgments (Foley $\&$ Foley, in press), these previous studies did not involve self/partner judgments. Therefore, it seemed important to assess item memory independent of source-monitoring judgments. In Experiment 2, we replicated the anagram conditions of Experiment 1 but assessed item memory by asking participants whether or not test items had been generated as solutions. In this test version, no reference was made to the source of the solutions.

\section{EXPERIMENT 2}

\section{Method}

Participants. Sixty undergraduates, none of whom participated in Experiment 1, participated in Experiment 2. As in Experiment 1, pairs of participants had the opportunity to win $\$ 25.00$.

Materials and Procedure. The materials and procedures were identical to those used in Experiment 1 except for the change introduced at test. All participants were eventually able to solve the anagram sets. Participants were surprised in this experiment with a recognition memory test on which they were asked to decide whether or not each test word had been a solution in the anagramsolving phase.

\section{Results}

Item memory. Item memory was computed as the proportion of old items correctly recognized as such. The mean proportions are reported in Table 4 as a function of source of solution (self or partner), anagram difficulty (easy or hard), and delay $(0,5$, or $15 \mathrm{sec})$. An ANOVA showed three significant effects. There was a main effect of source of solution $\left[F(1,56)=99.46, M S_{\mathrm{e}}=.01, p<\right.$ $.001]$ in which item memory was better for self-generated $(M=.85)$ than for partner-generated $(M=.69)$ solutions.
Table 4

Proportion of Old Items Called "Old" (Item Memory) in Experiment 2 as a Function of Delay, Source, and Anagram Difficulty

\begin{tabular}{|c|c|c|c|c|c|c|}
\hline \multirow{3}{*}{$\begin{array}{l}\text { Anagram } \\
\text { Difficulty }\end{array}$} & \multicolumn{6}{|c|}{ Delay } \\
\hline & \multicolumn{2}{|c|}{$0 \mathrm{sec}$} & \multicolumn{2}{|c|}{$5 \mathrm{sec}$} & \multicolumn{2}{|c|}{$15 \mathrm{sec}$} \\
\hline & $M$ & $S E$ & $M$ & $S E$ & $M$ & $S E$ \\
\hline \multicolumn{7}{|c|}{ Self-Generated } \\
\hline Easy & .84 & .03 & .79 & .05 & .82 & .03 \\
\hline Hard & .91 & .02 & .88 & .03 & .86 & .03 \\
\hline \multicolumn{7}{|c|}{ Partner-Generated } \\
\hline Easy & .45 & .04 & .78 & .03 & .85 & .03 \\
\hline Hard & .51 & .05 & .67 & .04 & .86 & .03 \\
\hline
\end{tabular}

There was a source $\times$ delay interaction $[F(2,56)=45.44$, $p<.001]$. Tukey-Kramer post hoc tests confirmed that this interaction was produced by a decrease in the memory advantage for self- over partner-generated solutions with increases in delay. This pattern was predicted, and it replicated the pattern reported in Experiment 1 for item memory.

Finally, there was a source $\times$ anagram difficulty interaction $\left[F(1,56)=8.84, M S_{\mathrm{e}}=.02, p=.004\right]$. This interaction emerged because although an item memory advantage was observed for solutions to hard anagrams $(M=$ $.88)$ in comparison with easy anagrams $(M=.82)$ for self-generated solutions, it was not observed for partnergenerated solutions $(M \mathrm{~s}=.69$ and .68 for easy and hard anagrams, respectively).

False positives. The proportion of new items called old (false positives) was computed as the proportion of new items for which an incorrect response of "old" was made during the source-judgment task. The mean proportions are reported in Table 2 as a function of delay $(0,5$, or $15 \mathrm{sec}$ ). As is clear from Table 2, false positive levels were quite low. However, a one-way ANOVA showed a main effect of delay $\left[F(2,56)=3.27, M S_{\mathrm{e}}=.01, p=.04\right]$ in which decreases in false positives were associated with increases in delay. Post hoc tests showed that the proportion of false positives observed in the 0 -sec delay condition was greater than those for the other two delay conditions, which did not differ from each other.

\section{Discussion}

The decrease in the generation effect for item memory across increases in delay that was found in Experiment 1 was again observed in Experiment 2. The pattern of false positives indicates that the differential effects of delay on item memory cannot be explained by a response bias toward reporting "partner," which would lead to an artificial inflation of item memory for partner-generated items. Thus, providing the participant with the opportunity to anticipate a partner's responses results in comparable item memory whether assessed indirectly through source judgments (Experiment 1) or directly (Experiment 2). In both experiments, the self-advantage decreased over the delay conditions because of an increase in memory for partner's solutions. 


\section{GENERAL DISCUSSION}

Within the context of partners alternating turns to solve a set of anagram problems, we predicted and found that opportunities to anticipate partners' responses affected both source accuracy and item memory. In Experiment 1, we tested the prediction that source accuracy (i.e., memory for the participant who was designated as the problem solver) would be affected by the opportunity to anticipate the solution. In particular, we expected that confusion would increase when there was a longer delay between the presentation of the anagram and the designation of the problem solver. The confusion was predicted to reduce source accuracy, and this was indeed the case, but only for easy anagrams.

Source accuracy about solutions to hard anagrams may have remained relatively constant over the delay conditions for several reasons. Presumably, the cognitive processes leading to the anticipation of solutions to hard anagrams would be more deliberative than those leading to the anticipation of solutions to easy ones. Additionally, failures to discover an anagram solution before learning that the partner was the solver, or discovering an anagram solution and feeling thwarted by the participant being named the solver, would be more likely to occur for hard anagrams. Thus, the relative kind or number of cognitive cues that are available to render source decisions presumably differed for easy and hard anagrams. Although the potential role of cues in relation to attempts (e.g., whether unsuccessful or thwarted) awaits future study, some aspects of our findings suggest that these other kinds of cues cannot fully account for the pattern of source accuracy in Experiment 1 . If the effects for anagram difficulty are tied to memory for cognitive operations that were initiated but thwarted, we should have observed an increase in source accuracy for solutions to hard anagrams over the delay conditions. This, however, was not the case.

As we mentioned earlier, source errors in sourcemonitoring tasks could arise from at least two kinds of recoding processes that occur during encoding. Anticipating solutions to partners' problems could be recoded as self-generations. In a sense, these anticipations are participants' own generations even when the answer was overtly expressed by their partners. However, source errors could also occur because observations of partners' responses - that is, hearing partners report their solutions aloud - could be recoded as self-generations (without requiring anticipation). Because source errors were observed in the 0 -sec delay condition in the absence of opportunities to anticipate, our findings point to the role of both kinds of recoding processes. Although the emphasis in the present studies is on the way in which recoding processes that are activated during encoding may lead to source errors, these inaccuracies may also arise from recoding processes that occur as an activity unfolds during retention intervals or testing (Foley et al., 2002).

We also predicted that item memory would be affected by the opportunity to anticipate solutions in ways that differed from the effects on item memory. In Experiments 1 and 2 , in the 0 -sec delay condition, item memory for self-generated solutions was better than item memory for partner-generated ones, illustrating the classic positive generation effect (see, e.g., Johnson et al., 1981; Slamecka \& Graf, 1978). Similarly, in the 0-sec delay condition, when new items were misrecognized as old, participants were more likely to report that these items were their partner's solutions rather than self-generated ones. Originally referred to as the "it had to be you" effect (Foley, Johnson, \& Raye, 1983; Johnson et al., 1981), this bias is thought to reflect participants' intuitions that what they do (or say) is more memorable than what they see (or hear) others do. Thus, if an item seems only somewhat familiar, participants would reason that someone else generated the item (in this case, the partner).

With increases in delay, however, the generation effect in item memory disappeared as a result of an enhancement in item memory for partners' solutions. With increases in delay allowing both participants time to view anagrams before one was identified as the solver, item memory for partners' responses increased. Thus, anticipating partners' responses (functionally, a form of self-generation) enhanced memory for the generation-effect "control" materials for the two delay conditions. Similarly, the low levels of false positives (Experiments 1 and 2) and the decrease in the tendency to report "partner" in response to new items in these delay conditions (Experiment 1) indicate that the enhancement effects for partners' solutions do not reflect general response biases.

Our item memory findings are not the first to note the importance of paying close attention to the basis of generation effects. Early interpretations of the generation effect suggested that the act of generating diverts attention such that control items are processed in a more shallow manner than they otherwise would be (Begg \& Snider, 1987; Slamecka \& Katsaiti, 1987). Such processing leads to decreases in memory for control materials, a view supported by substantial reductions in generation effects with the use of between-groups comparisons (see, e.g., Begg \& Snider, 1987). From this same perspective, the generation effect may reflect a reduction in memory for control items rather than an enhancement in memory for generated materials, leading one to expect that generating solutions to anagrams might encourage a similar inattentiveness to partners' generations. However, if generating solutions distracted attention from partners' items, we should have observed a reduction in item memory for partners' responses over increases in delay. We observed the opposite. Thus, our findings add to the demonstrations that the generation effect may be produced by variations in memory for control material rather than variations in memory for explicitly self-generated material. The contribution of the present findings is the evidence for the role of anticipation in memory for interactive exchanges. As such, these findings suggest that reports of generation-effect failures following interactive exchanges deserve careful scrutiny when testing predictions about the effects of cognitive operations (including anticipation) in memory.

Investigations of memory for interactive exchanges provided the impetus for the present studies because it was 
in these contexts that we noticed enhancement effects for partners' responses (e.g., Baker-Ward et al., 1990; Foley \& Ratner, 1998). Similarly, previous studies suggesting that anticipation contributes to confusion about the source of solutions have demonstrated the importance of the collaborative nature of the task (Foley \& Ratner, 1998; Ratner et al., 2002). Moreover, as we previously mentioned, in other kinds of interactive tasks the relationship between partners affects the expression of the generation effect. When friends are members of triads, generation-effect failures are reported (e.g., Baker-Ward et al., 1990). Consequently, we conceptualize the effects of anticipation in the present experiments as indications of the role of social and contextual variables in the expression of item and source memory. However, we also acknowledge that the effects of anticipation reported in Experiments 1 and 2 may be independent of the interactive nature of the task. That is, any context providing the opportunity to anticipate, whether interactive or not, might lead to similar effects. For example, in an anagram-solving task in which participants work alone to generate solutions to anagrams, advantages for self-generated and computer-presented solutions may be observed if participants have the opportunity to anticipate some other solutions (e.g., the computer presentation of solutions following anagram presentations after a brief delay). Although anticipation may play a role in noninteractive contexts, because of previous findings, we expect that its effects may be especially pronounced in more interactive ones. Fruitful directions for future research include the systematic comparison among alternative contexts for inducing anticipation to see whether social, interactive contexts are particularly sensitive to creating moments for anticipation.

\section{REFERENCES}

BaKer-Ward, L., Hess, T. M., \& Flannagan, D. A. (1990). The effects of involvement on children's memory for events. Cognitive Development, 5, 55-69.

BaYen, U. J., Murnane, K., \& Erdfelder, E. (1996). Source discrimination, item detection, and multinomial models of source monitoring. Journal of Experimental Psychology: Learning, Memory, \& Cognition, 22, 197-215.

BEGG, I., \& SNIDER, A. (1987). The generation effect: Evidence for generalized inhibition. Journal of Experimental Psychology: Learning, Memory, \& Cognition, 13, 533-563.

Brown, A. S., \& Murphy, D. R. (1989). Cryptomnesia: Delineating inadvertent plagiarism. Journal of Experimental Psychology: Learning, Memory, \& Cognition, 15, 432-442.

FoLEY, M. A., \& Foley, H. J. (in press). Source-monitoring judgments about anagrams and their solutions: Evidence for the role of cognitive operations information in memory. Memory \& Cognition.

Foley, M. A., Foley, H. J., Wilder, A., \& Rusch, L. (1989). Anagram solving: Does effort have an effect? Memory \& Cognition, 17, 755758.

Foley, M. A., Johnson, M. K., \& RaYe, C. L. (1983). Age-related changes in confusion between memories for thoughts and memories for speech. Child Development, 54, 51-60.

Foley, M. A., \& RATNER, H. H. (1998). Children's recoding memory for collaboration: A way of learning from others. Cognitive Development, 13, 91-108.

Foley, M. A., Ratner, H. H., \& Gentes, E. (2005, April). Walking in another's shoes: The role of perspective in memory for collaborative exchanges. Paper presented at the Biennial Meetings of the Society for Research in Child Development, Atlanta.

Foley, M. A., Ratner, H. H., \& House, A. T. (2002). Anticipation and source-monitoring errors: Children's memory for collaborative activities. Journal of Cognitive Development, 3, 385-414.

Foley, M. A., Ratner, H. H., \& PassalacQua, C. (1993). Appropriating the actions of another: Implications for children's memory and learning. Cognitive Development, 8, 373-401.

Gardiner, J. M., Ramponi, C., \& Richardson-Klavehn, A. (1999). Response deadline and subjective awareness in recognition memory. Consciousness \& Cognition, 8, 484-496.

GREENE, R. L. (1992). Human memory: Paradigms and paradoxes. Hillsdale, NJ: Erlbaum.

Greenwald, A. (1980). The totalitarian ego: Fabrication and revision of personal history. American Psychologist, 35, 603-618.

HICKS, J. L., \& MARSH, R. L. (2001). False recognition occurs more frequently during source identification than during old-new recognition. Journal of Experimental Psychology: Learning, Memory, \& Cognition, 27, 375-383.

Johnson, M. K., Hashtroudi, S., \& Lindsay, D. S. (1993). Source monitoring. Psychological Bulletin, 114, 3-28.

Johnson, M. K., RaYe, C. L., Foley, H. J., \& Foley, M. A. (1981). Cognitive operations and decision bias in reality monitoring. American Journal of Psychology, 94, 37-64.

JuRICA, P. J., \& ShIMAMURA, A. P. (1999). Monitoring item and source information: Evidence for a negative generation effect in source memory. Memory \& Cognition, 27, 648-656.

KinJo, H., \& SnODGRASS, J. G. (2000). Does the generation effect occur for pictures? American Journal of Psychology, 113, 95-121.

LANDAU, J. D., \& Marsh, R. L. (1997). Monitoring source in an unconscious plagiarism paradigm. Psychonomic Bulletin \& Review, 4, 265-270.

LiNDSAY, D. S., \& Johnson, M. K. (1991). Recognition memory and source monitoring. Bulletin of the Psychonomic Society, 29, 203-205.

Macrae, C. N., Bodenhausen, G. V., \& Calvini, G. (1999). Contexts of cryptomnesia: May the source be with you. Social Cognition, 17, 273-297.

Marsh, E. J., Edelman, G., \& Bower, G. H. (2001). Demonstrations of a generation effect in context memory. Memory \& Cognition, 29, 798-805

MARSH, R. L., \& Bower, G. H. (1993). Eliciting cryptomnesia: Unconscious plagiarism in a puzzle task. Journal of Experimental Psychology: Learning, Memory, \& Cognition, 19, 673-688.

MARSH, R. L., \& HICKS, J. L. (1998). Test formats change sourcemonitoring decision processes. Journal of Experimental Psychology: Learning, Memory, \& Cognition, 24, 1137-1151.

MARSH, R. L., \& LANDAU, J. D. (1995). Item availability in cryptomnesia: Assessing its role in two paradigms of unconscious plagiarism. Journal of Experimental Psychology: Learning, Memory, \& Cognition, 21, 1568-1582.

MARSH, R. L., LANDAU, J. D., \& HICKS, J. L. (1997). Contributions of inadequate source monitoring to unconscious plagiarism during idea generation. Journal of Experimental Psychology: Learning, Memory, \& Cognition, 23, 886-897.

MAYZNER, M. S., \& TrESSELT, M. E. (1958). Anagram solution times: A function of letter order and word frequency. Journal of Experimental Psychology, 56, 376-379.

Mulligan, N. W. (2001). Generation and hypermnesia. Journal of Experimental Psychology: Learning, Memory, \& Cognition, 27, 436450 .

MULLIGAN, N. W. (2004). Generation and memory for contextual detail. Journal of Experimental Psychology: Learning, Memory, \& Cognition, 30, 838-855.

MURNANE, K., \& BAYEN, U. J. (1996). An evaluation of empirical measures of source identification. Memory \& Cognition, 24, 417-428.

RATNER, H. H., Foley, M. A., \& GiMPERT, N. (2002). The role of collaborative planning in children's source monitoring errors and learning. Journal of Experimental Child Psychology, 81, 44-73. 
SlamecKa, N. J., \& Graf, P. (1978). The generation effect: Delineation of a phenomenon. Journal of Experimental Psychology: Human Learning \& Memory, 4, 592-604.

SLAMECKA, N. J., \& KATSAITI, L. T. (1987). The generation effect as an artifact of selective displaced rehearsal. Journal of Memory \& Language, 26, 589-607.

Srinivas, K., \& RoEDIger, H. L., III (1990). Classifying implicit memory tests: Category association and anagram solution. Journal of Memory \& Language, 29, 389-412.

StEFFENS, M. C., \& ERDFELDER, E. (1998). Determinants of positive and negative generation effects in free recall. Quarterly Journal of Experimental Psychology, 51A, 705-733.

TACONNAT, L., \& IsINGRINI, M. (2004). Cognitive operations in the generation effect on a recall test: Role of aging and divided attention. Journal of Experimental Psychology: Learning, Memory, \& Cognition, 30, 827-837.

TAYLOR, F. K. (1965). Cryptomnesia and plagiarism. British Journal of Psychiatry, 111, 1111-1118.
Thorndike, E. L., \& LORGE, I. (1944). The teacher's word book of 30,000 words. New York: Columbia University, Teachers College Press.

\section{NOTE}

1. The source test was scored in a second way as well, correcting for the differential false positive rates. This corrected measure had an impact on partner-generated items in the 0 -sec delay condition. Nevertheless, when using this corrected score, the pattern of source accuracy findings was the same as that reported for the traditional source measure. The source accuracy scores for self- and partner-generated solutions were .76 and .70 respectively, a nonsignificant difference.

(Manuscript received September 26, 2004; revision accepted for publication July 8,2005 .) 